Article

\title{
Fourier, Wavelet, and Hilbert-Huang Transforms for Studying Electrical Users in the Time and Frequency Domain ${ }^{\dagger}$
}

\author{
Vito Puliafito ${ }^{1}$, Silvano Vergura ${ }^{2, *} \neq$ and Mario Carpentieri ${ }^{2, \ddagger}$ \\ 1 Department of Engineering, University of Messina, I-98166 Messina, Italy; vpuliafito@unime.it \\ 2 Department of Electrical and Information Engineering, Polytechnic University of Bari, via E. Orabona 4, \\ I-70125 Bari, Italy; mario.carpentieri@poliba.it \\ * Correspondence: silvano.vergura@poliba.it; Tel.: +39-080-5963590; Fax: +39-080-5963410 \\ + This paper is an extended version of paper published in the International Conference: EEEIC 2016, Florence, \\ Italy, 7-10 June 2016. \\ $\ddagger$ These authors contributed equally to this work.
}

Academic Editor: Rodolfo Araneo

Received: 15 November 2016; Accepted: 3 February 2017; Published: 8 February 2017

\begin{abstract}
The analysis of electrical signals is a pressing requirement for the optimal design of power distribution. In this context, this paper illustrates how to use a variety of numerical tools, such as the Fourier, wavelet, and Hilbert-Huang transforms, to obtain information relating to the active and reactive power absorbed by different types of users. In particular, the Fourier spectrum gives the most important frequency components of the electrical signals, and the wavelet analysis highlights the non-stationarity of those frequency contributions, whereas the Hilbert-Huang transform, by means of the Empirical Mode Decomposition, provides a more complete spectrum of frequencies.
\end{abstract}

Keywords: non-stationary signal; time-frequency analysis; power quality; wavelet; Hilbert-Huang transform

\section{Introduction}

In the era of smart grids, the monitoring of power systems is needed for several purposes. It is used to detect failures in the electrical network, to underline the contributions of the distributed generators on the dynamic behavior of the whole system, and, more generally, to optimize the power distribution [1,2]. Nevertheless, the monitoring is not trivial, due to the massive use of non-linear loads and electronic-based equipment in residential, commercial, and industrial plants. In order to control the operation of the electrical lines, it is very important to implement and develop both on-line and off-line systems which are able to check the power fluctuations, related to the power absorbed by the numerous loads of each user. It is important to note that a disadvantage of this system is the large amount of data which has to be managed during the monitoring process. Even though several researchers have focused their attention on compacting the electrical information [3-5], a more detailed investigation on this state of the art process highlights that the compression of the signal waveform is not as mature as for speech, image, and video compression [6]. The complexity depends on the characteristics of the electrical signals of the modern equipment, which are related to the different loads (static, rotating, power electronics-based, and so on) and generators (deterministic-based, stochastic-based). The consequence of the diversity of the electrical loads induces the same electrical signals, which are nonlinear, non-stationary, and non-periodic.

In order to deal with this large amount of data and to manage the electrical signals, two main categories of mathematical tools, based on compression techniques, have been used: lossless techniques [7-9] and lossy algorithms [3,10-12]. Reference [6] reports a comparison of these tools 
(see Table I of [6]), based on the compression ratio and the distortion values. However, the appropriate choice of the technique to treat an electrical signal, is not trivial. In fact, as an example, if the electrical signal is linear and stationary, traditional mathematical tools, like the standard Fast Fourier transform (FFT), can be the correct analysis method. Vice versa, in the presence of electrical signals that deviate from linear and periodic behavior, different and more complex tools are necessary, in order to correctly characterize the electrical power behaviors. In recent years, the Wavelet Transform (WT) has been used increasingly often for several different issues related to power systems at every level. In [13], the authors study the modal parameters of power systems in order to analyze the origin of the small signal oscillations, thanks to the continuous WT that is able to detect the damping and frequency of the critical modes. Moreover, this approach uses the FFT to calculate the convolution integral of the continuous WT, in order to reduce the computational burden. The WT is also employed for the fault location and the power quality analysis in electric power systems [14], where a combination of several mathematical tools is proposed, on the basis of behavioral indexes, neural networks, statistics, and WT. In addition to the WT, the Hilbert-Huang Transform (HHT) is an effective tool for studying the non-stationary phenomena of power systems. In [15], the authors propose an iterative HHT to study the power quality waveforms in the presence of a sudden transition. Moreover, to discriminate the correct instant, they also use the Symbolic Aggregate ApproXimation. HHT, together with the discrete WT, is also used to analyze the power system areas with coherent generator groups, therefore in no-fault conditions [16].

In this framework, the WT [12-17] and the Empirical Mode Decomposition (EMD) deriving from the calculation of the HHT $[18,19]$ were also used with success, in order to determine the properties of non-linear, non-stationary, and non-periodic electrical signals of passive or active distribution lines (i.e., in the absence or presence of renewable energy sources, respectively). These tools, together with the FFT, allow a complete analysis of those signals, both in time and frequency domain.

The WT, specifically, evidences the non-stationarity of the signals $[17,20]$. In the distribution lines, WT was used to analyze the absorbed power in anomalous conditions [12], and to characterize different typologies of electrical users [21].

In addition, the HHT-EMD produces a description of the behavior of different kinds of signals that are typically nonlinear [22-25], overcoming the limitations of the FFT analysis, which is able to quantitatively describe signals with linear, stationary, and periodic characteristics. The efficiency of the HHT analysis of electrical power signals has already been tested, by comparing the HHT-based results and the FFT-based results, both for seasonal periods [26] and for long periods [19]. For the sake of completeness, when both the non-linearity and the non-stationarity are present, the Duffing equation [27] is an effective tool for investigating the electrical behavior of distribution lines.

This paper, in contrast to the previous literature and [21], which describes the FFT and WT analysis of the active power, presents a complete and deep methodology, based on the FFT, WT, and HHT-EMD analysis of both active and reactive power. The dataset derives from measured data of distribution lines, feeding different typologies of users (private residences, groceries, marketing retails, general services, restaurants). We also want to emphasize that, whereas in our previous work [19] we used FFT, WT, and HHT-EMD tools to study the different electrical behaviors of the active and passive distribution lines, here we use the same tools to highlight the different electrical behavior of end-users belonging to different typologies, with the aim of characterizing the end-user.

The paper is organized as follows. Section 2 describes the theoretical background, illustrating both the WT and the HHT; data under investigation are presented in Section 3; Section 4 discusses the numerical results after having processed our dataset by means of FFT, WT, and HHT; lastly, Section 5 is devoted to the conclusions.

\section{Theoretical Framework}

In this Section, we present the numerical methods used for the analysis of both the active and reactive power of the electrical users. 
In particular, we firstly introduce the WT used to characterize signals in the time-frequency domain, pointing out the non-stationary behaviors. Secondly, the EMD based on the HHT formalism is presented, in order to correctly describe the non-linear behavior of these kinds of signals.

\subsection{Wavelet Transform}

The continuous wavelet transform $W(u, s)$ of a time-domain signal $x(t)$ is a linear function, defined as:

$$
W(u, s)=\frac{1}{\sqrt{s}} \int_{-\infty}^{\infty} x(t) \psi *\left(\frac{t-u}{s}\right) d t
$$

where $s$ and $u$ are the scale and translation parameters of the mother wavelet $\psi(t)$, that defines the wavelet family function as:

$$
\psi_{u, s}(t)=\frac{1}{\sqrt{s}} \psi\left(\frac{t-u}{s}\right)
$$

The following complex Morlet mother wavelet has been utilized in this paper:

$$
\psi_{u, s}=\frac{1}{\sqrt{s \pi f_{B}}} e^{j 2 \pi f_{C}\left(\frac{t-u}{s}\right)} e^{-\left(\frac{t-u}{s}\right) / f_{B}}
$$

where $f_{B}$ and $f_{C}$ are two characteristic parameters. After setting the dimension $N$ of the scale set $\{s\}$, the optimal scale set $\{s\}_{i=1, \ldots, N}$ for the WT have been found, adopting the method reported in Reference [28-30].

\subsection{HHT and EMD}

Given a time-domain signal $X(t)$, the HHT-based approach provides a calculation of the Hilbert transform $Y(t)=H\{X(t)\}$ :

$$
Y(t)=\frac{1}{\pi} P \int_{-\infty}^{\infty} \frac{X\left(t^{\prime}\right)}{t-t^{\prime}} d t^{\prime}
$$

where $P$ represents the principal value. Instead, the Hilbert complex extension of $X(t)$ is constituted by the analytical signal associated to $X(t), Z(t)=X(t)+j Y(t)=X(t)+j H\{X(t)\}=a(t) e^{j \theta(t)}$, where $j$ is the imaginary unit, whereas the amplitude $a(t)$ and the phase $\theta(t)$ are represented by the following equations: $a(t)=\left[X^{2}(t)+Y^{2}(t)\right]^{\frac{1}{2}}$ and $\theta(t)=\arctan \frac{Y(t)}{X(t)}$, respectively. Considering the application of the stationary phase method to the Fourier transform $F(\omega)$ of $Z(t)$, it yields $\omega(t)=d \theta(t) / d t$, where $\omega(t)$ is the instantaneous angular frequency [31]. By applying the methodology proposed in [18], and finalized to the EMD, the signal $X(t)$ can be considered as a finite collection of single characteristic intrinsic modes, defined as Intrinsic Mode Functions (IMFs), i.e., $X(t)=\sum_{l=1}^{N} c_{l}(t)+r_{N}(t)$, where $c_{l}(t)$ are the time-dependent IMFs with variable amplitude and $r_{N}(t)$ is the non-oscillatory residue term, due to the sifting process. The number of oscillations of the IMFs is related to their order, reducing the first, as the second increases. This effect is due to the algorithm employed for the iterative extractions of the IMFs. As it will be shown later, each iteration of EMD generates a new signal, obtained as the difference between the original signal and the mean value of the envelope curves, of its local maximum and minimum values.

For each $\operatorname{IMF} c_{l}(t)$, having a characteristic angular frequency $\omega_{l}(t)$, the complex representation can be written as:

$$
z_{l}(t)=c_{l}(t)+j \frac{1}{\pi} P \int_{-\infty}^{\infty} \frac{c_{l}\left(t^{\prime}\right)}{t-t^{\prime}} d t^{\prime}=a_{l}(t) e^{j \theta_{l}(t)}
$$

where $a_{l}(t)$ is the amplitude of $z_{l}(t)$, while $\theta_{l}(t)=\int \omega_{l}(t) d t, l=1,2, \ldots, N$. 
Furthermore, the amplitude $X(t)$ of an electrical signal expanded in a Fourier series, thus highlighting the IMF components, has the following form:

$$
X(t)=\operatorname{Re}\left\{\sum_{l=i}^{N} z_{l}(t)\right\}=\operatorname{Re}\left\{\sum_{l=i}^{N} a_{l}(t) e^{j \theta_{l}(t)}\right\}=\operatorname{Re}\left\{\sum_{l=i}^{N} a_{l}(t) e^{2 \pi j \int} f_{l}(t)\right\}
$$

where $R e$ is the real part, and $f_{l}(t)=\omega_{l}(t) / 2 \pi$ is the instantaneous frequency of the $l$-th IMF, whereas the residue $r_{N}(t)$ is neglected, for instance when it is a monotonic function, which does not allow any extraction of IMFs.

In this paper, the proposed methodology is applied to two time-domain signals: the active power $P(t)$ and the reactive power $Q(t)$. Consequently, the Hilbert transform defined in Equation (4), and the Fourier representation of Equation (6), are expressed in terms of either $P(t)$ or of $Q(t)$. Equation (6) is the Hilbert amplitude spectrum $H(\omega, t)$, also known as the Hilbert spectrum.

\section{Description of the Data and Coherence Degree}

The dataset under investigation is constituted by the active and reactive powers absorbed by 25 different users, grouped into five different typologies (restaurants, groceries, marketing retails, general services, private residences). The sampling time of the measurements was fixed at $15 \mathrm{~min}$, and the overall period of recording was three months, from the 1st of December, 2014, to the 28th of February, 2015, covering 90 consecutive days with 96 samples per day $(90 \times 96=8640$ samples recorded per user). In exceptional cases (less than $0.5 \%$ ), the power measurements were not available in the dataset, thus we connected the external values via interpolation. In this regard, after comparing different kinds of interpolation, the average of the values observed in similar circumstances (same hours in different days) turned out to be the most reliable method.

The first step concerned the time-domain analysis of the measured data, in order to extract the main features of the active power absorbed by the users, such as the maximum power and the typical timing of the power request. This analysis allowed an evaluation of the similarity level of the five users belonging to the same user typology and, then, whether it could be possible to average their behaviors, in order to identify the sample user of that typology, or to consider whether one user is representative of each typology. Successively, in order to estimate the likeness between users of the same category, we calculated the spectral coherence between the power signals of each couple of users, for the same typology. The time-frequency coherence of two signals, $x(t)$ and $y(t)$, is a real-valued function, defined as:

$$
C_{x y}(f)=\frac{\left|S_{x y}(f)\right|^{2}}{S_{x}(f) S_{y}(f)}
$$

where $S_{x y}(f)$ is the cross-spectral density between $x(t)$ and $y(t)$, and $S_{x}(f)$ and $S_{y}(f)$ are the autospectral densities of $x(t)$ and $y(t)$, respectively. The values of the coherence always range from zero to one [32,33]. Table 1 shows the coherence values obtained by the Equation (7), including the average coherence of each user. The less coherent users for each typology are: restaurant \#4, grocery \#1, marketing retail \#2, general services \#1, and private residence \#5.

Finally, our proposed time-frequency analysis was completed. We will first plot the time-domain power signals of a user for each typology, over the whole data acquisition period. Next, we report on the frequency-domain analysis, including the frequency spectrum obtained by means of FFT, the WT scalogram, and the HHT analysis. 
Table 1. Coherence Values between Active Power Signals.

\begin{tabular}{cccccc}
\hline \multirow{2}{*}{ Users } & \multicolumn{5}{c}{ Coherence } \\
\cline { 2 - 5 } & Restaurants & Groceries & Marketing Retails & General Services & Private Residences \\
\hline$\# 1-\# 2$ & 0.9463 & 0.9365 & 0.8773 & 0.6653 & 0.9866 \\
$\# 1-\# 3$ & 0.9661 & 0.9595 & 0.9655 & 0.6798 & 0.9952 \\
$\# 1-\# 4$ & 0.9116 & 0.9310 & 0.8879 & 0.7033 & 0.9913 \\
$\# 1-\# 5$ & 0.9375 & 0.9594 & 0.9382 & 0.7058 & 0.9225 \\
$\# 2-\# 3$ & 0.9464 & 0.9609 & 0.9008 & 0.9961 & 0.9821 \\
$\# 2-\# 4$ & 0.9324 & 0.9548 & 0.9302 & 0.9812 & 0.9870 \\
$\# 2-\# 5$ & 0.9426 & 0.9825 & 0.9036 & 0.9718 & 0.9114 \\
$\# 3-\# 4$ & 0.9194 & 0.9504 & 0.9005 & 0.9837 & 0.9048 \\
$\# 3-\# 5$ & 0.9441 & 0.9684 & 0.9281 & 0.9751 & 0.9320 \\
$\# 4-\# 5$ & 0.9300 & 0.9505 & 0.9112 & 0.9819 & 0.9739 \\
& & & Average Coherence for Each User & 0.9668 \\
$\# 1$ & 0.9404 & 0.9466 & 0.9172 & 0.6886 & 0.9675 \\
$\# 2$ & 0.9419 & 0.9587 & $\underline{0.9030}$ & 0.9036 & 0.9746 \\
$\# 3$ & 0.9440 & 0.9598 & 0.9237 & 0.9087 & 0.9177 \\
$\# 4$ & 0.9234 & 0.9467 & 0.9075 & 0.9125 & 0.9087 \\
\hline 5
\end{tabular}

\section{Results and Discussion}

\subsection{Temporal Analysis of the Active and Reactive Power}

From the coherence analysis results, we have chosen one user which is the most representative of each typology. Figure 1a reports the active power absorbed by the user restaurant \#2 in the trimester, whereas Figure $1 \mathrm{f}$ is a zoomed-in view of a 4-day interval, from a Friday to a Monday of February. As it can be seen, this specific user mainly absorbs power during two specific periods, lunch and dinner time, respectively. Moreover, it is almost always closed on Sundays. The diagram points out when the absorbed power is very low, corresponding to the closing days. The Christmas holidays are evidenced by the longest closing period.
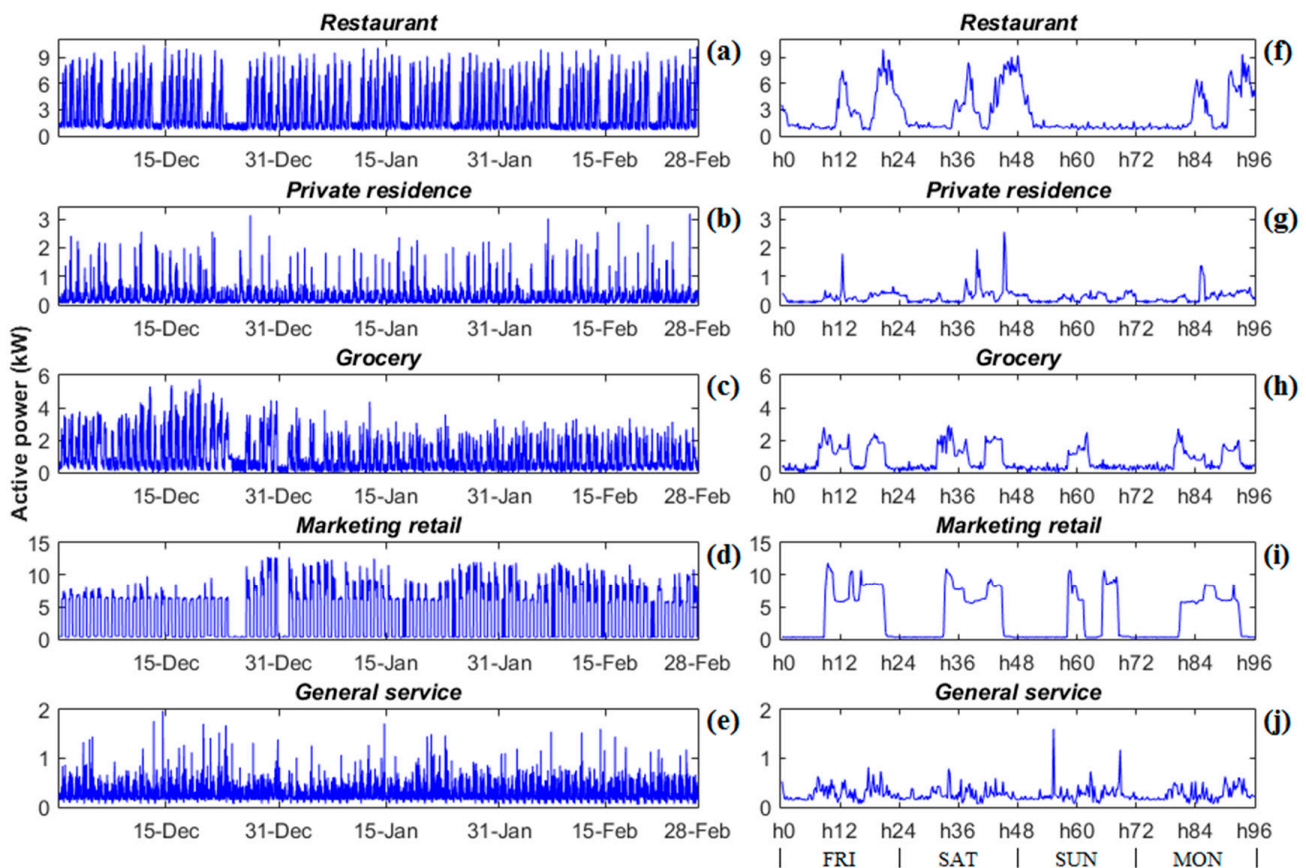

Figure 1. Example of temporal evolution of the active power absorbed by (a) a restaurant; (b) a private residence; (c) a grocery; (d) a marketing retail establishment; (e) a general service, for the whole measurements campaign. (f-j) Active power absorbed by the same users in a four day interval, from Friday 13th to Monday 16th of February. 
User \#1 is the reference for the category Private residence \#1, and Figure $1 \mathrm{~b}$ diagrams its active power (supply contract of $3 \mathrm{~kW}$ ). A detailed forecasting of the absorbed power is impossible to obtain during the day, nevertheless, it is mainly concentrated during the day hours. As you can see, there is no appreciable difference in the absorbed power, with respect to the Christmas period, as occurred in the previous case.

Figure 1c reports the power absorbed by the user grocery \#3, which has a $6 \mathrm{~kW}$ supply contract. The absorbed power is concentrated in two periods, the morning and the afternoon opening times. Sunday is the closing day. In addition, the time evolution of the power highlights, once again, a very low power request during the Christmas holidays.

Figure $1 \mathrm{~d}$ shows the power absorbed by user \#1 of the category marketing retail, with a $10 \mathrm{~kW}$ supply contract. The absorbed power is regular during the whole daytime, specifically from 9 a.m. to 9 p.m. It is evident that the retail establishment is usually open on Sundays, until the lunch break. Finally, the retail is closed during Christmas day and New Year's day.

Figure 1e regards general service \#2 (supply contract of $1.5 \mathrm{~kW}$ ). The absorbed power is largely variable during the day, and it depends on the particular typology of the electrical load, including elevators, lights in common spaces, and so on, and therefore, the requested power is not predictable during the day.

Figure 2 shows a similar study of the reactive power, for the same users analyzed above.

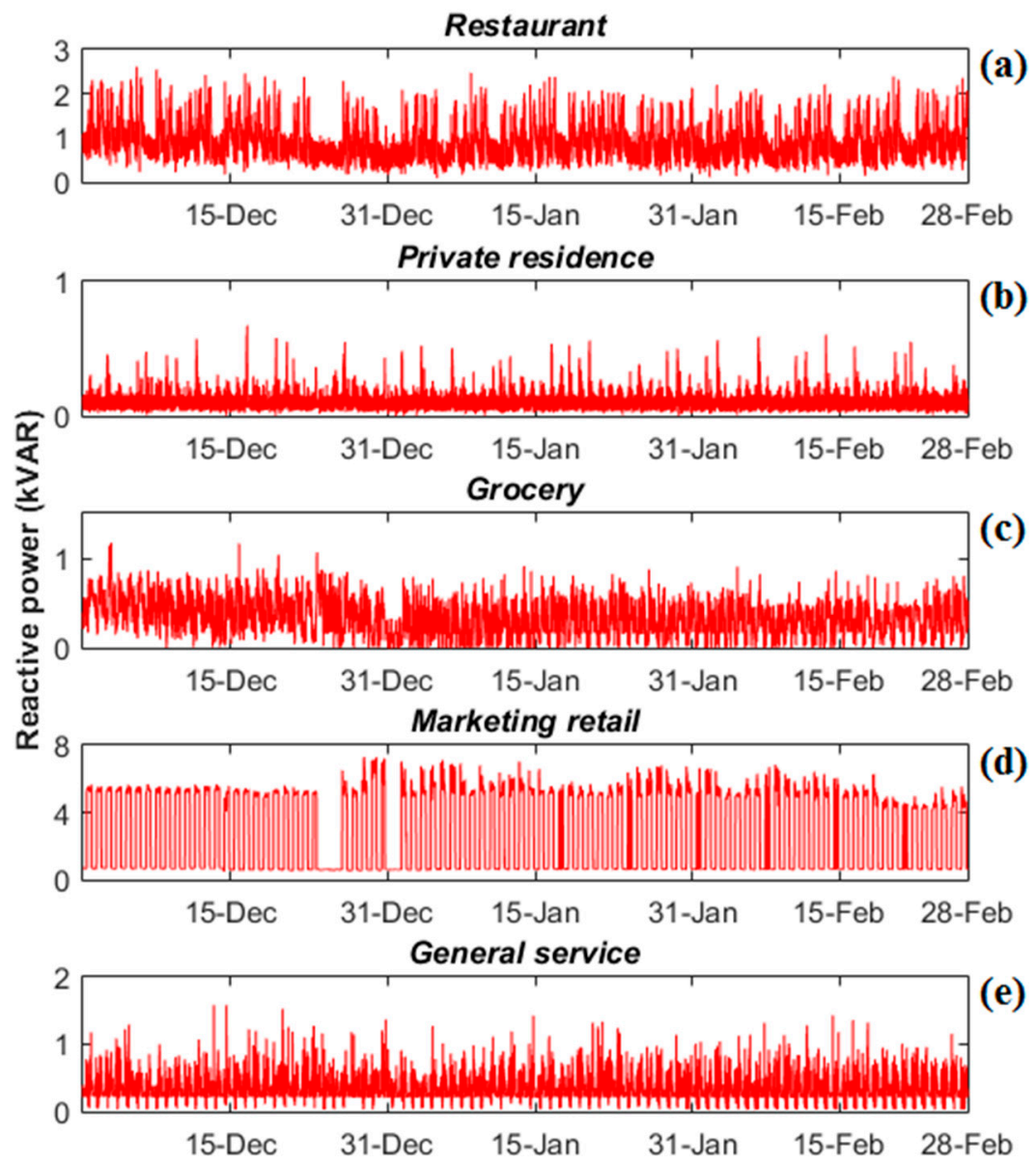

Figure 2. Example of temporal evolution of the reactive power requested by (a) a restaurant; (b) a private residence; (c) a grocery; (d) a marketing retail establishment; (e) a general service, for the whole measurements campaign.

As expected, in the private residences, the reactive power contribution is very low, because there are loads absorbing a very low quantity of reactive power. Vice versa, restaurants and marketing retail 
establishments produce the most interesting users for the analysis of the reactive power, because their typical electrical loads are transformers and motors. In both cases, you can observe a power request during the day hours, whereas small values of reactive power are cyclically requested by the restaurant during the night.

\subsection{FFT Analysis}

The Fourier spectrum of the first considered user (restaurant \#2) shows the most important frequency components, which are at $f_{1}=1.157 \times 10^{-5} \mathrm{~Hz}, f_{2}=2.315 \times 10^{-5} \mathrm{~Hz}$, and $f_{3}=3.472 \times 10^{-5} \mathrm{~Hz}$, corresponding to 24,12 , and $8 \mathrm{~h}$, respectively, and are named $\mathrm{P}_{1}, \mathrm{P}_{2}$, and $\mathrm{P}_{3}$, respectively, in Figure 3a. The Fourier spectrum of private residence \#2 (see Figure $3 \mathrm{~b}$ ) highlights the contributions of $P_{1}, P_{2}, P_{3}, P_{4}\left(f_{4}=4.630 \times 10^{-5} \mathrm{~Hz}\right.$, corresponding to $\left.6 \mathrm{~h}\right), P_{5}\left(f_{5}=5.787 \times 10^{-5} \mathrm{~Hz}\right.$, corresponding to $4.75 \mathrm{~h})$, and $P_{6}\left(f_{6}=6.944 \times 10^{-5} \mathrm{~Hz}\right.$, corresponding to $\left.4 \mathrm{~h}\right)$.
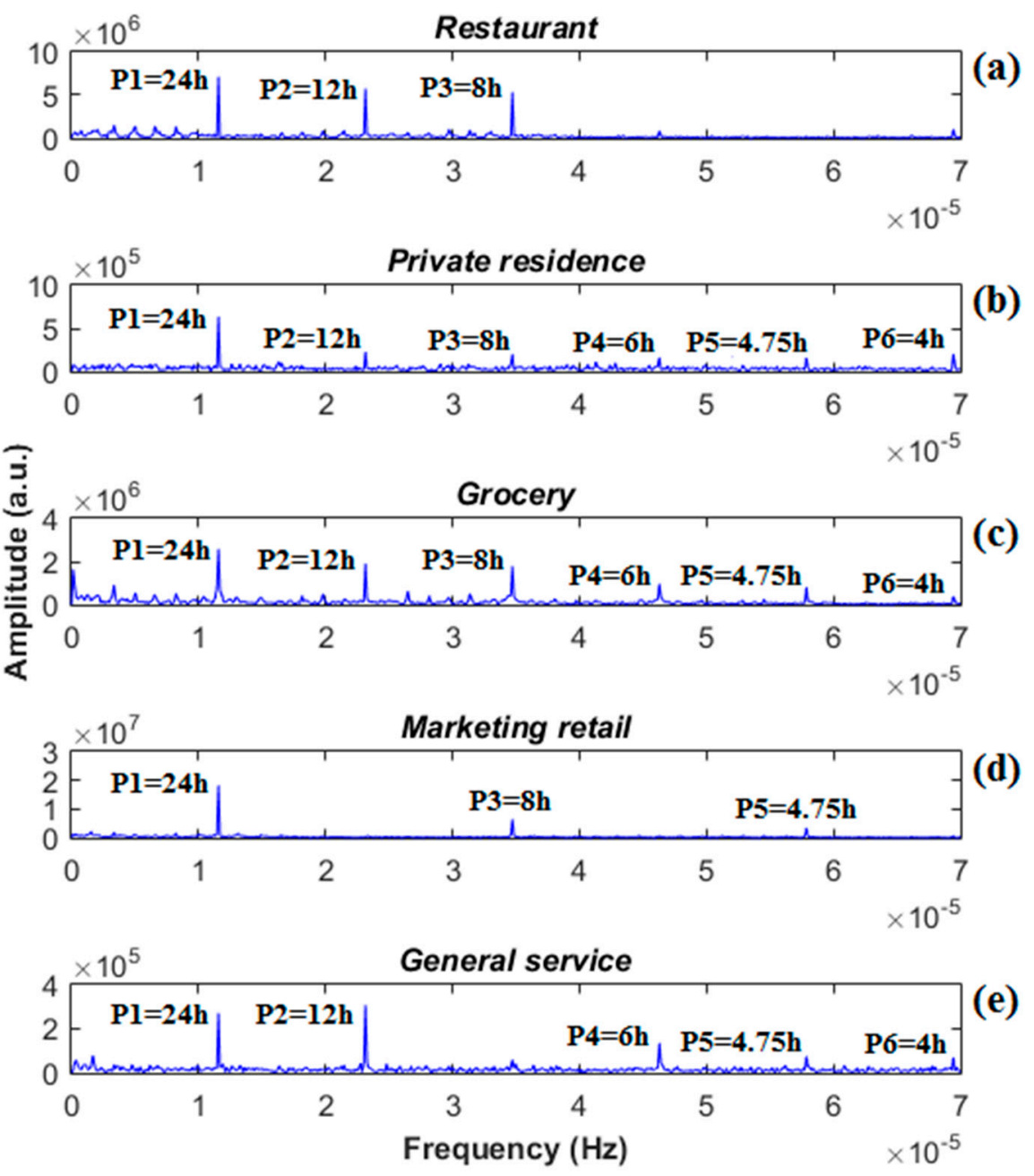

Figure 3. Fourier spectrum of the active power with the peaks highlighted: (a) restaurant; (b) private residence; (c) grocery; (d) marketing retail establishment; (e) general service.

Figure $3 c$ represents the Fourier spectrum of grocery \#3, where the same peaks of the previous case are detected, without the peak $P_{6}$. In the case of marketing retail \#1, the Fourier spectrum only contains the frequency components, $P_{1}, P_{3}$, and $P_{5}$ (see Figure $3 \mathrm{~d}$ ), related to the odd components of the Fourier spectrum. Frequency contributions of the general service $\# 2$ (Figure 3 e) include $P_{1}, P_{2}, P_{4}, P_{5}$, and $P_{6}$.

When observing the Fourier analysis of the reactive power (see Figure 4), it becomes evident that the main frequency modes are the same for the related user of the active power spectrum, when the 
absorbed active and reactive powers are comparable. In our case, the only user which has a reactive power that is much lower than the active one, is the private residence (see Figures $1 b$ and $2 b$ ). In fact, the spectrum of the active and reactive power contains different frequency peaks, all of the harmonics of the spectrum for the active power, and only the first harmonic for the reactive power.
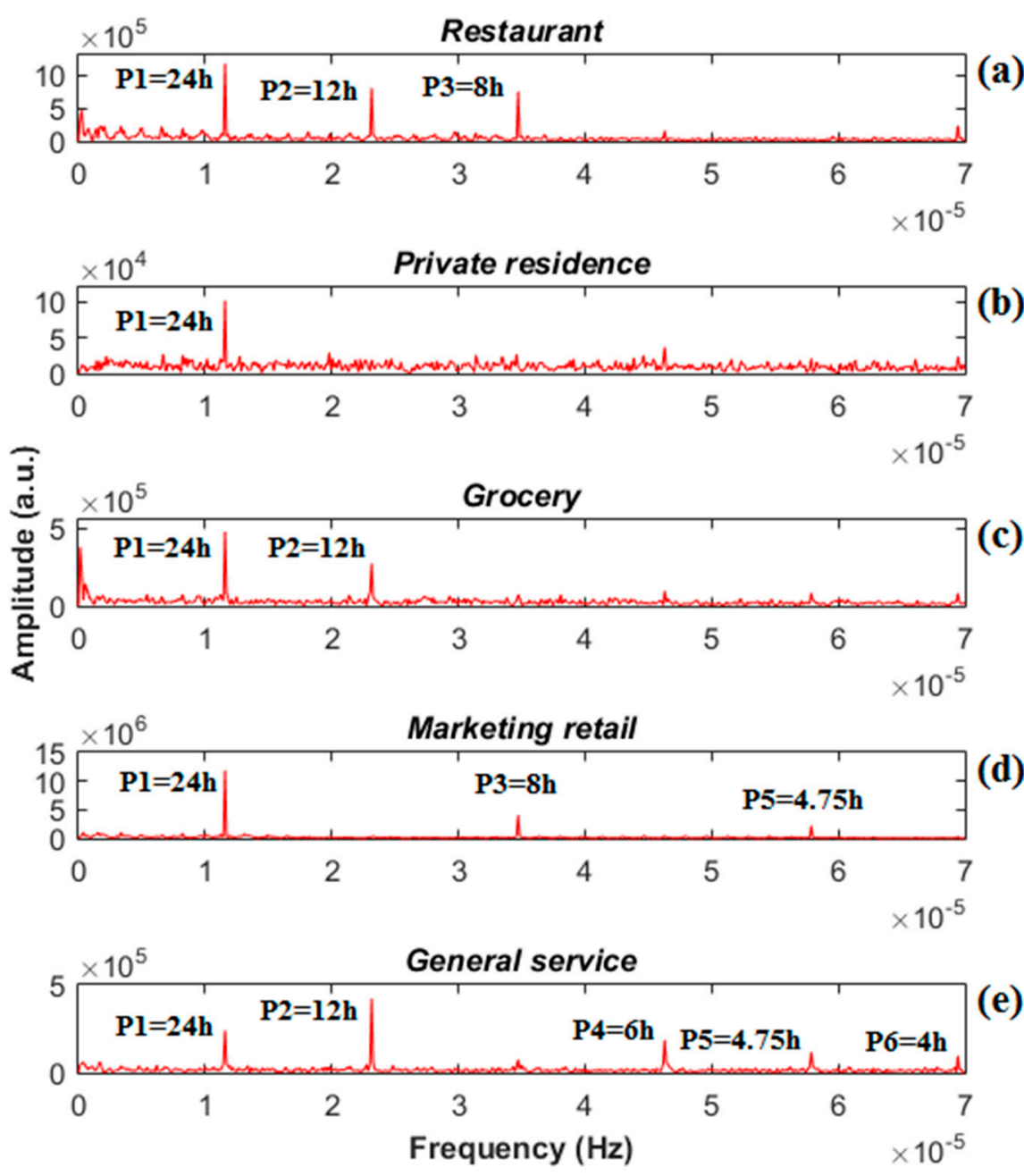

Figure 4. Fourier spectrum of the reactive power with the peaks highlighted (a) restaurant; (b) private residence; (c) grocery; (d) marketing retail establishment; (e) general service.

\subsection{Wavelet Analysis}

The WT analysis is an effective mathematical method for studying the non-stationary behavior of the electrical signals.

Figure 5 shows the WT scalogram of the active power for each user under study. In particular, Figure 5a is related to the restaurant, where $P_{1}$ and $P_{2}$ appear during the Christmas time, thus resulting in quasi-stationary modes. $P_{3}$ takes into account the closing days, highlighting an intermitting excitation mode. Figure $5 \mathrm{~b}$ reports the $\mathrm{WT}$ analysis for the private residence, where it is observed that, apart from the stationary mode $P_{1}$, the other harmonics are less significant and discontinuous. Concerning the grocery user, Figure $5 \mathrm{c}$ shows that the relevant modes, $P_{1}$ and $P_{2}$, appear during the whole trimester, while the other modes are only present in December. Figure $5 \mathrm{~d}$ points out that, in the case of the marketing retail, $P_{1}$ is the only stationary mode, and instead, $P_{3}$ and $P_{5}$ are low power and non-stationary modes. Finally, Figure 5 e illustrates that the first two peaks of the general service are quasi-stationary modes, while $P_{4}$ is only present in the final acquisition period. 
Figure 6 reports the WT analysis for the reactive power of the same users discussed above. In the case of the restaurant, the scalogram shows the stationarity of the $P_{1}$ mode; $P_{2}$ is quasi-stationary, whereas $P_{3}$ is attenuated with respect to the corresponding active power peak. Concerning the grocery, the $P_{1}$ mode is stationary; however, it is weak during the Christmas period, which is related to a different work organization of the user. Marketing retail and general service show frequency modes similar to those obtained in the active power analysis during the whole period. As expected, the WT scalogram of private residence confirms that the reactive power is almost negligible.
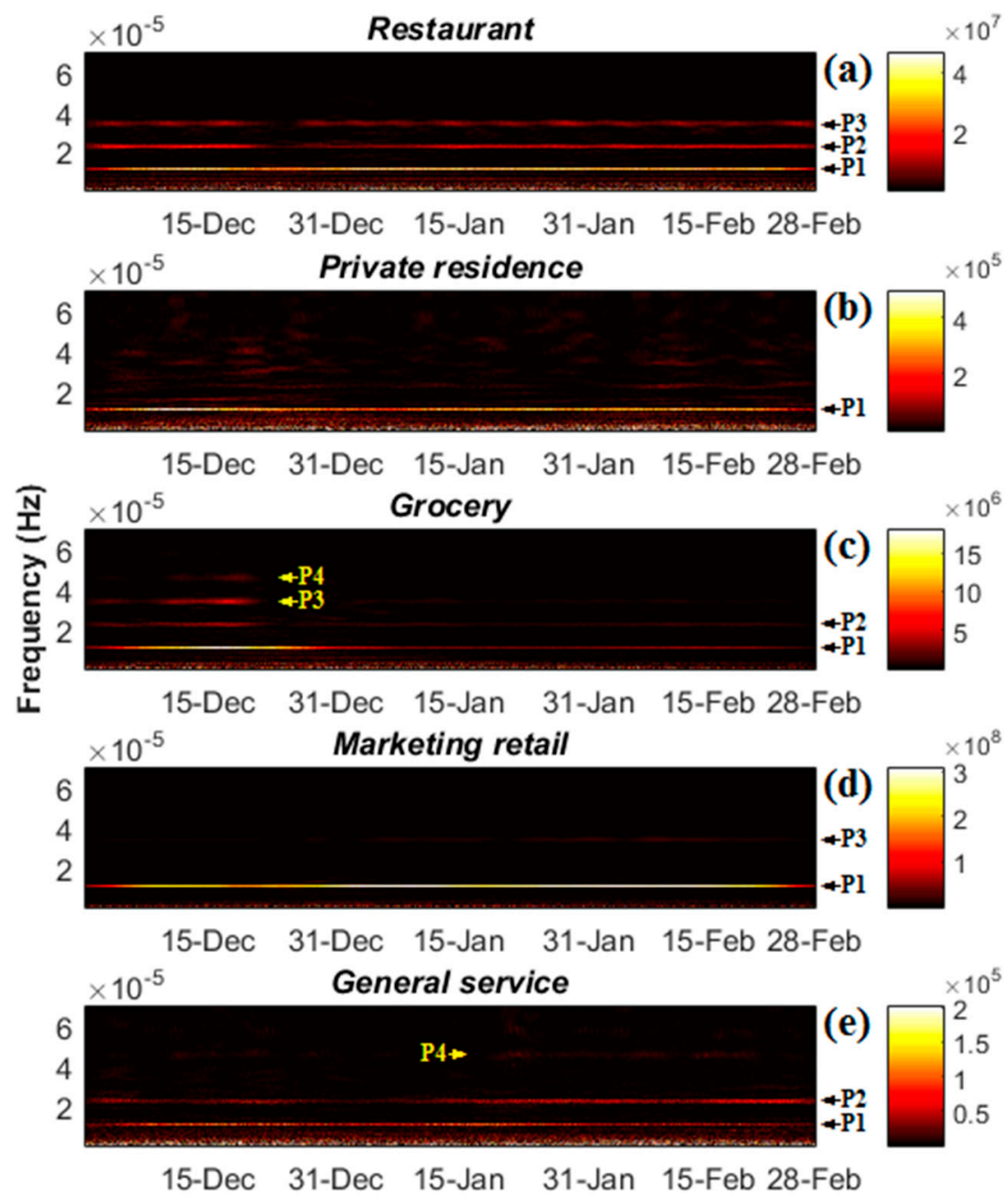

Figure 5. Wavelet scalogram of the active power for (a) a restaurant; (b) a private residence; (c) a grocery; (d) a marketing retail establishment; (e) a general service. 

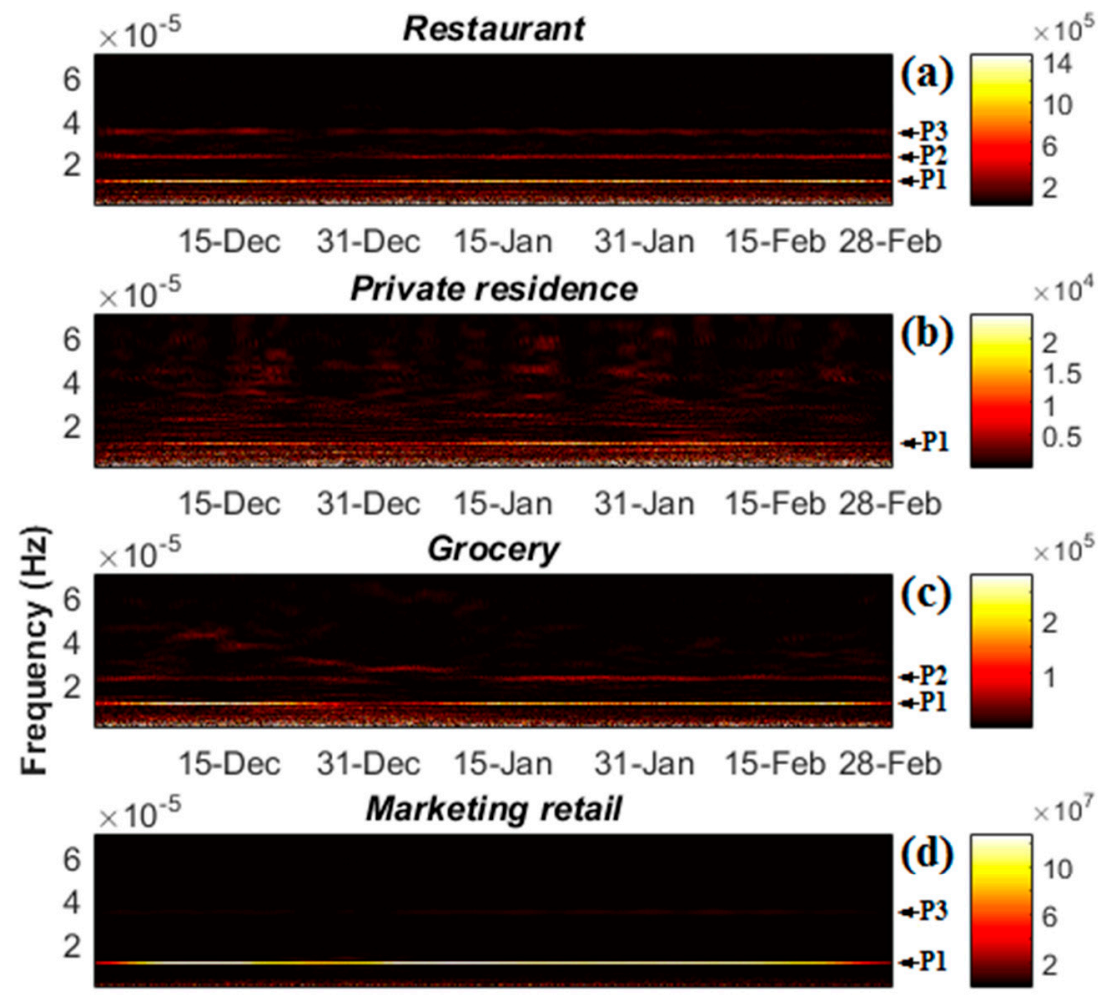

15-Dec 31-Dec 15-Jan 31-Jan 15-Feb 28-Feb

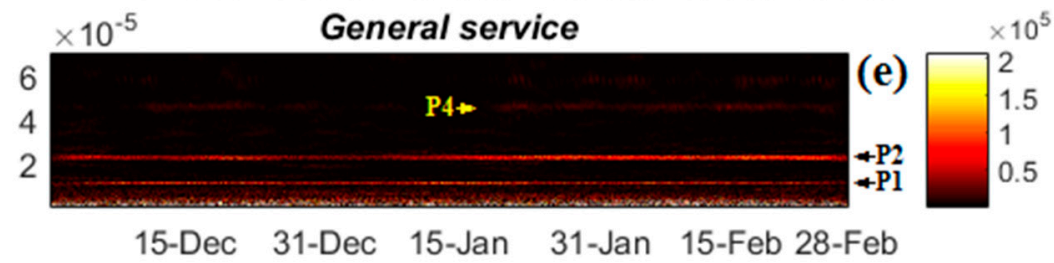

Figure 6. Wavelet scalogram of the reactive power for (a) a restaurant; (b) a private residence; (c) a grocery; (d) a marketing retail establishment; (e) a general service.

\subsection{HHT Analysis}

In order to provide an extensive analysis of the tools available to treat these particular electrical signals, characterized by the non-linear and non-stationary behavior, this section presents the results carried out by applying the HHT transform to one of the previous users (restaurant). Similar results are found for the other users (for the sake of brevity, not shown here).

In agreement with the EMD analysis on the basis of the HHT technique, the active power signal is decomposed into $12 \mathrm{IMFs}$ (Figure 7, left column), whereas the reactive power one is decomposed into 11 IMFs (Figure 7, right column), for the whole sampling period. The IMFs are organized into a decreasing frequency order, due to the higher number of temporal oscillations of the signal, with respect to each successive iteration. The evidence that the active power shows a larger number of IMFs than the reactive power, is due to its higher non-linear behavior of the electrical signal under investigation. The frequencies of the intermediate IMFs, in our case \#4-6, are very close to the ones found by performing the FFT analysis; all of the other IMFs have frequencies that do not correspond to those of the FFT harmonics.

Figure $8 \mathrm{a}, \mathrm{b}$ show the frequency peaks of the IMFs for active and reactive power, respectively. By comparing the HHT spectrum with the frequencies computed by the FFT, we observe that the HHT frequency range is much larger than the one found by using the FFT, and this means that the electrical signal of both the active and reactive power, is not periodical and non-linear. In fact, when comparing the excited modes found in the FFT (see Figures 3a and 4a) and HHT spectrum, we observe 
that only the frequencies of the IMFs \#4-6 for the active power and the reactive power, overlap with the frequency peaks computed by FFT analysis.

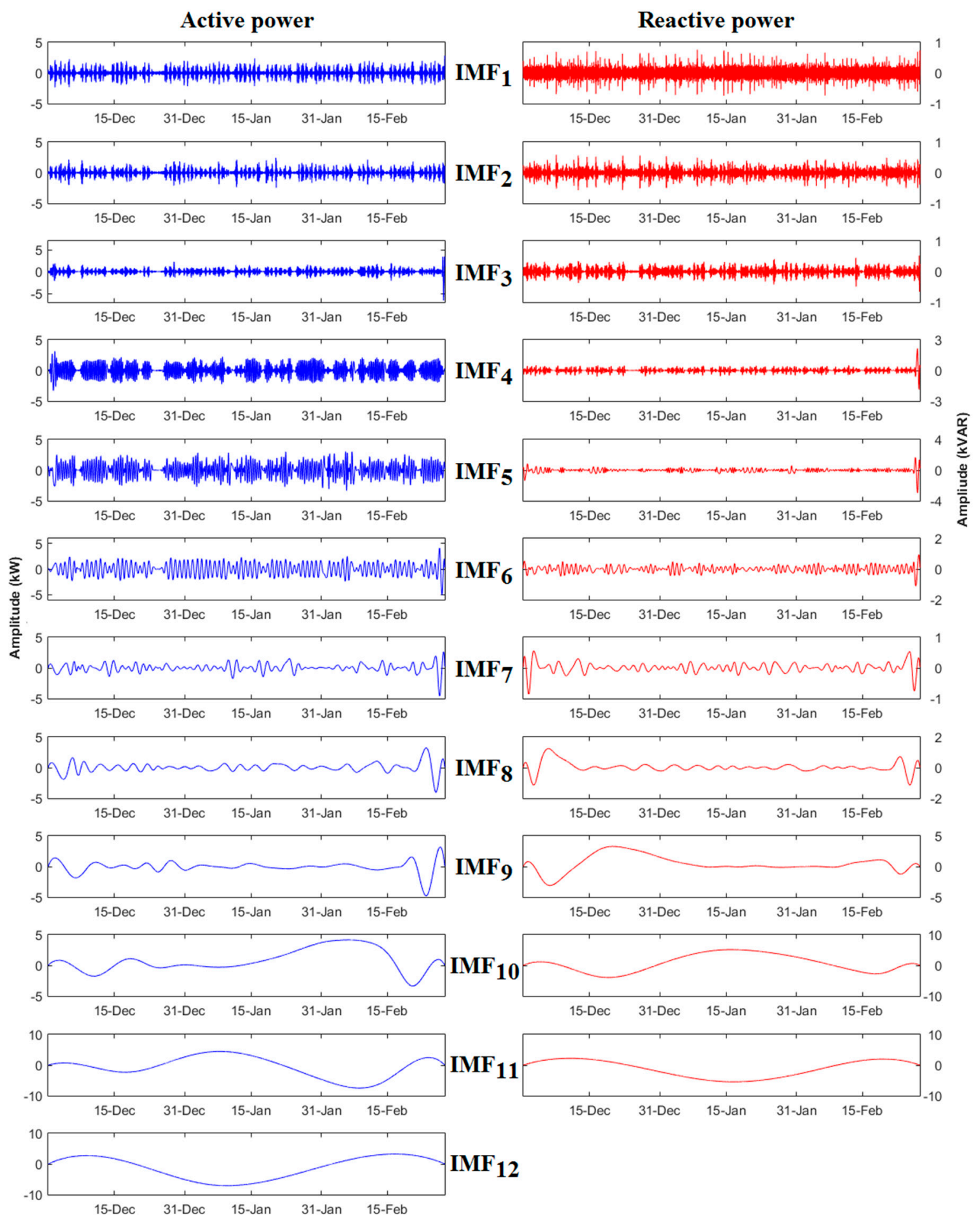

Figure 7. Time-domain amplitude of the IMFs of a restaurant user for the active power (left column) and reactive power (right column). 

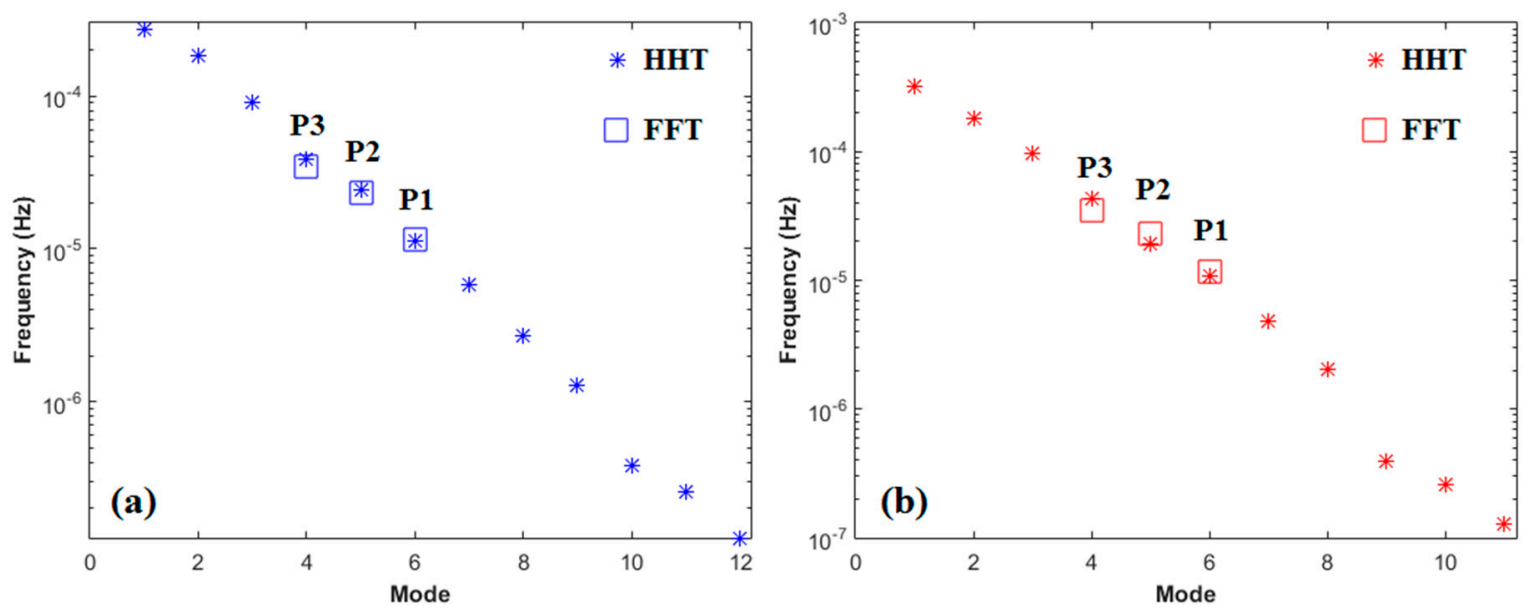

Figure 8. HHT-EMD (stars) and FFT (square) frequencies related to a restaurant user for (a) the active power and (b) the reactive power.

\subsection{Discussion}

From the results reported above, we find out that the FFT provides general information on the frequency behavior of both the active and reactive power. It is worth noting that the FFT is not the best method for studying non-stationary and non-linear signals acquired over a long period. In fact, the Fourier spectrum does not provide information on the time instant when the mode is excited, because it is time-independent. In the presence of non-stationary signals, the WT analysis overcomes this problem, because it computes the Fourier spectrum for different temporal windows. For example, Figures 5 and 6 show that the frequency modes, for both the active and reactive power, are often not stationary during the whole period (three months), and the amplitude of the frequency peaks is not always constant.

FFT and WT are not able to extract all the frequency modes of the non-linear electrical signals, because they are covered by the noise and/or other frequency modes. From our analysis, the HHT transform has turned out to be a very useful tool for detecting all the frequency modes present in the active and reactive power of the case-studies. In fact, the information coming from the HHT is richer than that from the FFT, including a larger number of frequency components. Specifically, in the studied cases, for both active and reactive power, the modes of the FFT spectrum are contained in the central region of the HHT spectrum. The higher and lower frequency modes are not revealed when using the FFT and WT analyses, whereas the HHT spectrum includes the working modes at high and low frequency regimes, which are not revealed by the FFT analysis. The extraction of all the components is very important for several reasons. For example, many electrical loads contain magnetic parts, whose losses are frequency-dependent; therefore, it is only possible to correctly evaluate the losses if all the frequency components are known. Again, the magnetic parts of the electrical loads can be strongly nonlinear, and thus, the effects of the high frequency components cannot be negligible. Other similar examples of nonlinear and nonstationary signals can support the importance of the proposed analysis. Nonetheless, computational requirements for performing WT and HHT-EMD analysis are not significantly higher than the ones needed for a FFT calculation, and for this reason, the proposed methodology is effective and fast.

\section{Conclusions}

The analyses discussed above give a complete picture of the effectiveness of the different tools used to characterize the electrical signals. The different peculiarities of each analysis tool (FFT, WT, and HHT) imply that the choice of an appropriate method for the electrical signal to be studied is not trivial. 
In conclusion, this paper shows that the HHT-EMD tool allows an extraction of the characteristic properties of the electrical signals, which are not found using the FFT and WT analyses; moreover, it can be used for both active and reactive powers. Finally, the frequency spectrum calculated via HHT-EMD is wider, with respect to the spectrum identified by means of the FFT and WT analyses. The reason for this is attributable to the non-periodical behavior of the electrical signals, connected to their strong deviation from linearity.

Acknowledgments: The paper has been written within the framework of the project "RES NOVAE-Reti, Edifici, Strade-Nuovi Obiettivi Virtuosi per l'Ambiente e l'Energia". This project is supported by the Italian University and Research National Ministry (MIUR) and competitiveness program that Italy is developing to promote "Smart Cities Communities and Social Innovation".

Author Contributions: V.P. performed the numerical analyses, and prepared the figures. S.V. and M.C. interpreted the results. All of the authors contributed to the writing of the manuscript.

Conflicts of Interest: The authors declare no conflict of interest.

\section{References}

1. Samuelsson, O.; Hemmingsson, M.; Nielsen, A.H.; Pedersen, K.O.H.; Rasmussen, J. Monitoring of power system events at transmission and distribution level. IEEE Trans. Power Syst. 2006, 21, 1007-1008. [CrossRef]

2. Vergura, S.; Acciani, G.; Amoruso, V.; Patrono, G.E.; Vacca, F. Descriptive and inferential statistics for supervising and monitoring the operation of PV plants. IEEE Trans. Ind. Electr. 2009, 56, 4456-4464. [CrossRef]

3. Ning, J.; Wang, J.; Gao, W.; Liu, C. A wavelet-based data compression technique for smart grid. IEEE Trans. Smart Grid 2011, 2, 212-218. [CrossRef]

4. IEEE Recommended Practice for Monitoring Electric Power Quality; IEEE Standard 1159-2009; IEEE: New York, NY, USA, 2009.

5. Huang, S.J.; Jou, M.-J. Application of arithmetic coding for electric power disturbance data compression with wavelet packet enhancement. IEEE Trans. Power Syst. 2004, 19, 1334-1341. [CrossRef]

6. Tcheou, M.P.; Lovisolo, L.; Ribeiro, M.V.; da Silva, E.A.B.; Rodrigues, M.A.M.; Romano, J.M.T.; Diniz, P.S.R. The compression of electric signal waveforms for smart grids: State of the art and future trends. IEEE Trans. Smart Grid 2014, 5, 291-302. [CrossRef]

7. Gerek, O.N.; Ece, D.G. Compression of power quality event data using 2D representation. Electr. Power Syst. Res. 2008, 78, 1047-1052. [CrossRef]

8. Kraus, J.; Tobiska, T.; Bubla, V. Looseless encodings and compression algorithms applied on power quality datasets. In Proceedings of the 20th International Conference and Exhibition on Electricity Distribution-Part 1, Prague, Czech Republic, 8-11 June 2009.

9. Kraus, J.; Stepan, P.; Kukacka, L. Optimal data compression techniques for smart grid and power quality trend data. In Proceedings of the 15th International Conference on Harmonics and Quality of Power (ICHQP), Hong Kong, China, 17-20 June 2012.

10. Qing, A.; Hongtao, Z.; Zhikun, H.; Zhiwen, C. A compression approach of power quality monitoring data based on two-dimension DCT. In Proceedings of the 2011 Third International Conference on Measuring Technology and Mechatronics Automation (ICMTMA), Shanghai, China, 6-7 January 2011.

11. Zhang, M.; Li, K.; Hu, Y. A high efficient compression method for power quality applications. IEEE Trans. Instrum. Meas. 2011, 60, 1976-1985. [CrossRef]

12. Vergura, S.; Siracusano, G.; Carpentieri, M.; Finocchio, G. A nonlinear and non-stationary signal analysis for accurate power quality monitoring in smart grids. In Proceedings of the 3rd Renewable Power Generation Conference (IET-RPG), Naples, Italy, 24-25 September 2014.

13. Hosseini, S.A.; Amjady, N.; Velayati, M.H. A Fourier Based Wavelet Approach Using Heisenberg's Uncertainty Principle and Shannon's Entropy Criterion to Monitor Power System Small Signal Oscillations. IEEE Trans. Power Syst. 2015, 30, 3314-3326. [CrossRef]

14. Bíscaro, A.A.; Pereira, R.A.F.; Kezunovic, M.; Mantovani, J.R.S. Integrated Fault Location and Power-Quality Analysis in Electric Power Distribution Systems. IEEE Trans. Power Deliv. 2016, 31, 428-436. [CrossRef]

15. Afroni, M.J.; Sutanto, D.; Stirling, D. Analysis of Nonstationary Power-Quality Waveforms Using Iterative Hilbert Huang Transform and SAX Algorithm. IEEE Trans. Power Deliv. 2013, 28, 2134-2144. [CrossRef] 
16. Avdaković, S.; Bećirović, E.; Nuhanović, A.; Kušljugić, M. Generator Coherency Using the Wavelet Phase Difference Approach. IEEE Trans. Power Syst. 2014, 29, 271-278. [CrossRef]

17. Goswami, J.C.; Chan, A.K. Fundamentals of Wavelets. In Theory, Algorithms and Applications, 1st ed.; Wiley-Interscience: Hoboken, NJ, USA, 2000.

18. Huang, N.E.; Shen, Z.; Long, S.R.; Wu, M.C.; Shih, H.H.; Zheng, Q.; Yen, N.-C.; Tung, C.C.; Liu, H.H. The empirical mode decomposition and the Hilbert spectrum for nonlinear and non-stationary time series analysis. Proc. R. Soc. Lond. A 1998, 454, 903-995. [CrossRef]

19. Vergura, S.; Zivieri, R.; Carpentieri, M. Indices to study the Electrical Power Signals in Active and Passive Distribution Lines: A Combined Analysis with Empirical Mode Decomposition. Energies 2016, 9, 211. [CrossRef]

20. Hong, Y.Y.; Chen, Y.Y. Placement of power quality monitoring using enhanced genetic algorithm and wavelet transform. IET Gener. Transm. Distrib. 2011, 5, 461-466. [CrossRef]

21. Vergura, S.; Carpentieri, M.; Puliafito, V. A time-frequency analysis of electrical users by means of Fourier and Wavelet transforms. In Proceedings of the 16th International Conference on Environment and Electrical Engineering (EEEIC 2016), Florence, Italy, 7-10 June 2016.

22. Oberlin, T.; Meignen, S.; Perrier, V. An alternative formulation for the empirical mode decomposition. IEEE Trans. Signal Process. 2012, 60, 2236-2246. [CrossRef]

23. Mandic, D.P.; Rehman, N.U.; Wu, Z.; Huang, N.E. Empirical mode decomposition-based time-frequency analysis of multivariate signals. IEEE Signal Process. Mag. 2013, 30, 74-86. [CrossRef]

24. Huang, N.E.; Kunot, A. An optimization based EMD scheme. J. Comput. Appl. Math. 2013, 240, $174-183$. [CrossRef]

25. Wang, T.; Zhang, M.; Yu, Q.; Zhang, H. Comparing the applications of EMD and EEMD on time-frequency analysis of seismic signal. J. Appl. Geophys. 2012, 83, 29-34. [CrossRef]

26. Vergura, S.; Zivieri, R.; Carpentieri, M. Seasonal signal analysis of distribution lines in smart grids via hilbert-huang transform. In Proceedings of the 15th International Conference on Environment and Electrical Engineering (EEEIC2015), Roma, Italy, 10-13 June 2015.

27. Zivieri, R.; Vergura, S.; Carpentieri, M. Analytical and numerical solution to the nonlinear cubic Duffing equation: An application to electrical signal analysis of distribution lines. Appl. Math. Model. 2016, 40, 9152-9164. [CrossRef]

28. Siracusano, G.; Finocchio, G.; La Corte, A.; Consolo, G.; Torres, L.; Azzerboni, B. Non stationary magnetization dynamics driven by spin transfer torque. Phys. Rev. B 2009, 79, 104438. [CrossRef]

29. Siracusano, G.; Torres, L.; Puliafito, V.; Finocchio, G. A generalized tool for accurate time-domain separation of excited modes in spin-torque oscillators. J. Appl. Phys. 2014, 115, 17D108. [CrossRef]

30. Puliafito, V.; Siracusano, G.; Azzerboni, B.; Finocchio, G. Self-modulated soliton modes excited in a nano-contact spin-torque oscillator. IEEE Magn. Lett. 2014, 5, 3000104. [CrossRef]

31. Huang, N.E.; Wu, Z.; Long, S.R.; Arnold, K.C.; Chen, X.; Blank, K. On instantaneous frequency. Adv. Adapt. Data Anal. 2009, 1, 177-229. [CrossRef]

32. White, L.B.; Boashash, B. Cross spectral analysis of non-stationary processes. IEEE Trans. Inf. Theory 1990, 36, 830-835. [CrossRef]

33. Winter, R.G.; Steinberg, A.M.; Attwood, D. Coherence, AccessScience, McGraw-Hill Education. Available online: http:/ / accessscience.com/content/146900 (accessed on 8 February 2017).

(C) 2017 by the authors; licensee MDPI, Basel, Switzerland. This article is an open access article distributed under the terms and conditions of the Creative Commons Attribution (CC BY) license (http:/ / creativecommons.org/licenses/by/4.0/). 